Historic, Archive Document

Do not assume content reflects current scientific knowledge, policies, or practices. 


\section{RETAIL LIST}

\section{Spring, 1927}

\section{GLADIOLUS BULBS}

Choicest Varieties from World's Leading Originators

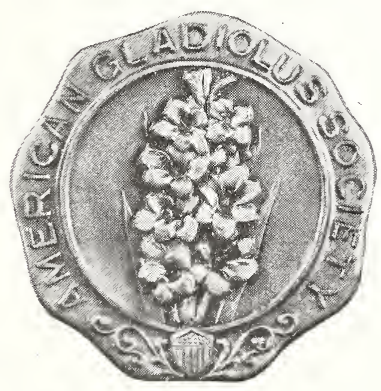

American Gladiolus Society Silver Medal

New York, August 1924

\section{B. HALSEY SPENCER}




\section{INTRODUCTION}

The Gladiolus has been my favorite flower since I was a youngster. My first collection contained varieties forgotten to-day, yet choice and beautiful in their time. No flower has been improved as much or increased in popularity as rapidly. In this list of 250 varieties I present for your consideration the Choicest Varieties of the World's Leading Originators.

You will find they cover every conceivable color; combination; the best and the latest introductions; in fact a list of quality glads equal to that of any other Grower.

I grew over 700 different named varieties last season besides those in mixture and my seedlings. Some are not listed, as orders at the Field or Shows took all available bulbs. Others amount of stock is too limited. Some of them will go to improve my mixture. And many will be discarded all together to make room for the new things as they are introduced, of which only the best will be retained. 


\section{PRIZE WINNERS}

My only entrance for prize competition last season was at the show of the CONNECTICUT GLADIOLUS SOCIETY at Hartford, Conn. where my blooms won the Presidents Cup, the highest award, also FIRST for the finest display of Gladiolus and other awards.

Very complimentary remarks have come from those who saw my exhibition bed in Forest Park, Springfield, Mass.; my private exhibit at CROFT'S The FLORIST in Springfield, The Eastern States Exposition and my largest display at LANE the FLORIST in Hartford, Conn. where a two days exhibit was extended to five to accomodate the many visitors and 1500 spikes were shown covering 80 different high class varieties.

Every spike shown at these exhibits were taken from the field as they came. Not a spike was grown for exhibition.

\section{VISITORS.}

Visitors are always welcome. This past season in addition to my Longmeadow field I had a five acre piece in Suffield, Conn. where I had my show garden, visited by hundreds of people from all sections of the country. GLADIOLUS SUNDAY was a big success and will be an annual affair. There was a constant line from early morning until dark making it impossible to wait on them all.

\section{PRIGES.}

I have endeavored to make my prices coincide with those of reliable Growers which are commensurate with high quality bulbs and variety. I may be higher in a few cases, lower in others, but what ever you pay I GUARANTEE SATISFACTION. Bulbs will be Full size, clean, healthy, husky. True to name. Should an error occur which may happen, no matter how careful one may be, let me know at once. IT will be made right. I make it a point to give a customer the very best, just as I would demand myself if I was in their position.

\section{EXTRAS.}

ALL ORDERS ARE CASH for which extra count will be given and $10 \%$ additional in bulbs of my choosing. If received before MARCH 1st, I promise nice things.

On $\$ 10.00$ orders or over I will give a bulb of Marnia and small bulb of Rose Mist or equivalent when those are gone.

On orders of $\$ 15.00$ and up other specials will be given. NO order accepted less than $\$ 1.00$. Prices Pre-paid.

On orders of $\$ 100.00$ I will give a bulb of ORCHID LADY, my Prize Winning Seedling. desire.

ORDER EARLY if you want to be sure to get what you

I have fine testimonials that there is not room to publish. Orders have come from the same customers the five years I have been in the Gladiolus business. Try an order. Compare and Report.

\section{5\% DISCOUNT TO A. G. S. MEMBERS}

\section{SPEGIALS \\ ORGHID LADY}

My seedling. Queen of the lavenders.

Winner of the A. G. S. Silver Medal. Orchid Lavender, with silvery sheen and touch of cream in the throat.

The center of attraction at Hartford Exhibit, $\$ 15.00$ each two for $\$ 25.00$.

FROSTY. A light rose red Prim. flaked gray, each .25.

SNOWBIRD. A very attractive white Prim with yellow throat, each .25. 


\section{MIXTURES.}

LONGMEADOW MIXTURE. This is composed of all named varieties of high quality. I know what many mixtures are as I have bought them and thrown them away after seeing the mess they contained. I Guarantee this mixture will please. I am always sold out of large bulbs every season. .75 per Doz. $\$ 5.00$ per $100, \$ 40.00$ per 1000 .

Special Mixture. Made up from named varieties, $\$ 7.50$ per 100 .

Many customers have reordered this again as they found it an "excellent lot of Bulbs."

EXTRA SPECIAL. Made up of higher priced named varieties, $\$ 10.00$ per 100 . Bulbs put in this if bought under name would cost around $\$ 15.00$.

MEADOWVALE MIXTURE. This is a primulinus mixture of named varieties. If you want color in your garden in many shades and exquisite hues get some of this. It is HIGH CLASS in every way. .50 per Doz., $\$ 3.50$ per $100, \$ 28.00$ per 1000 .

\section{AMERIGAN GLADIOLUS SOCIETY}

Join now. An organization devoted to the advancement of the Gladiolus. You will receive the BULLETIN monthly, devoted solely to the Gladiolus and $5 \%$ discount from Growers. Send $\$ 2.00$ and I will see you are enrolled, and allow discount on your order.

\section{THE FLOWER GROWER.}

One of the finest Flower Magazines published. Monthly $\$ 2.00$ per year. If you are not a subscriber you really should be. Send me $\$ 2.00$ and I will see that you are enrolled for a years subscription and I will send you free 25 Gladiolus Bulbs with out cost.

\section{GLADIOLUS}

Flaming sword of the garden, Tempered with sun and due, Forged by the Master Dream-Smith, Joan might have carried you!

Pierced by your bright-edged beauty, Conquered and bleeding I fall.

Slay me not with your glory,-

I, too, hear Voices Call. 


\section{PRICE LIST}

ADORATION $(\mathbf{K})$. Large ruffled saffron. Fine $\$ 25$ ADRIATIG $(\mathbf{K})$. Wine blue, red throat...................... $\quad .10$ ADA DE POY (S). Light orange pink .................... .25 ADELINE KENT (D). Ruffled light pink ..................... .15 ALBANIA (K p). Very fine white................................. .15 ALMA GLUCK (G). Fine peach red............................. 15 ALTON (K). Ruffled orange. A winner........................ .15 ANNA EBERIUS (D). Popular purple...................... .15 ANTHONY B. KUNDERD (K). Cream and Pink. R. .25 ARISTOCRAT (B). Yellowish salmon. Fine............ 1.50 AGATHA (K). Deep peach Blossom pink................... .15 ANTHONY ZONKER (K). Large ruffled salmon pink with blotch. Very fine.

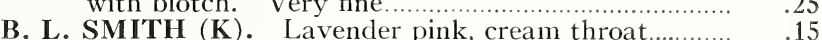

BEVIER (K). Salmon rose, creamthroat ........................ 15

BENGAL TIGER (P). Red, tiger striped.......................

BIG BLACK (K). Large fine black red....................... . 25

BLANGHE BOLLINGER (K). Old rose striped lighter 2.00 BREAK OF DA Y (B). Wild rose pink ....................... 3.50

BLUE LILY $(\mathbf{K})$. Light violet blue ........................... 15

BUTTERBOY $(\mathbf{K})$. Tall fine early yellow .................... .20

BLUE ISLE (K). Lilac blue. Very fine ..................... .20

BUCKEYE $(\mathbf{K})$. Fine old rose pink .......................... 25

GATHERINE COLEMAN (G). Large salmon rose pink $\quad .60$ GAPT. BOYN'TON. Lavender blue on white. Blotch.. $\quad .30$

GARDINAL PRINGE (K). Clear cardinal red............. 1.50

GARMEN SYLVA (Dec.). One of the best whites....... .15

GIRCE (K). Early mahogany red. Fine..................... 15

CLASSIC (K). Shell pink. Blotch........................... 10

GRIMSON GLOW (Bet.). Very fine. Large................ 08

GLAREMONT (S). Light yellow. Deep yellow throat .75

GRINKLES (K). Deep peach blossom pink .............. .25

GRUSADER (F). Deep maroon. Excellent ................. 4.00

CAPT. MILES S'TANDISH (F). No better red. Self.. .75

COPPER BRONZE $(\mathbf{K})$. Very attractive prim............ 2.00

CORUNNA (K). Red, black throat bordered white....... .15

GRUENTUS SUPERBA (Bet.). Late red. Fine......... .50

CAPELLA $(\mathbf{K})$. Tall red xxxx prim.......................... 15

DIANA $(\mathbf{Z})$. A real fine red .................................... 10

D's AMERICAN BEAUTY. Creamthroat, many open .15

DREAM (K). Ruffled salmon red. Large................... .35

DORRITT $(\mathbf{K})$. Large showy lavender pink............... $\quad .20$

DORO'THY McKIBBON (K). Ruffled rose pink. Fine .10

DR. F. E. BENNETT (D). Large orange scarlet.......... .75

DR. VAN FLEET (K). Tall rose. Yellow throat........ .15

DR. ELKINS $(\mathbf{K})$. Large white, purple throat................ .25

DR. J. H. NEELEY (K). Tall white tinted pink.......... .15

DR. R. T. JAGKSON (F). Very dark velvety red....... 10

DR. NELSON SHOOK $(\mathbf{K})$. Deep Tyrean rose............ 1.50

DON JUAN (K). Similar to M. Foch with blue tint on edges.....

E. J. SHA YLOR (K). Strong deep violet rose ............ 08

E. G. HILL (K). True pink, yellow in throat.............. .25

ELIZABETH TABOR $(\mathbf{H})$. Early rose pink, red blotch .20

EMILE AUBRUN (L). Very large copperybronze, red 3.00

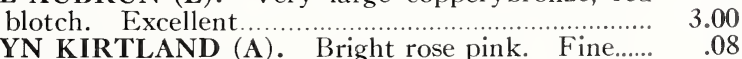

$\begin{array}{llcc}\text { EVELYN KIRTLAND (A). Bright rose pink. } & \text { Fine..... } & .08 \\ \text { ELKHART }(\mathbf{K}) . & \text { Violet purple deeper throat. } & \text { Fine..... } & .50\end{array}$

EXCELLO (K). Soft pink, dark blotch...................... .15

EXQUISITE $(\mathbf{K})$. American beauty rose. A beauty.... .25

EUROPA. An old white but a good one ........................ .15

E. B. WILLIAMSON (K). Light purple prim................ .25

ETHL YN (F). Very fine orange prim........................ 30

ELDORA (Kp). Buff pink .........................................

FAIR COLUMBIAN (K). Tall showy lilac..................... 15

FERN K YLE (K). Large cream yellow, very good........ .30

FLORA $(\mathbf{H})$. Best low priced yellow.......................... Sold

FREDA (S). Large geranium pink............................. 20 
FORTUNA (F). Gorgeous large pink

GERTRUDE ERREY (Aus.). Bright pink tinged salmon.....

GLENDALE (Da.). Like the best red cyclamen....

GERALDINE FARRAR (D). Pale lavender violet....... Sold

GIAN'T MYRTLE (K). Fine pale rose pink............... .25

GIANT NYMPH $(\mathbf{C})$. Very large light salmon ros?....... .25

GETTYSBURG (Dec.). Excellent dark red............... .75

GLADDIE BOY (B). Orange pink and yellow prim..... 2.00

GLORIANA (Bet.). Golden salmon, yellow throat...... 2.00

GLORIOSA (Bet.). Yellow strippled light red............. .25

GOLDEN DREAM (Gr.). Clear dark yellow grand....... 10.00

GOLDEN BUTTERFLY $(\mathbf{K})$. Grand canary yellow..... . .25

GOLDEN FRILLS (K). Ruffled light yellow.............. 4.00

GOLDEN EAGLE (A). Early clear yellow, fine........... 12.00

GOLD (Dec.). Good refined yellow............................... .15

GOLDEN MEASURE (Kel.). Tall strong deep yellow.. $\quad .20$

GOLD ELSIE (F). Real deep yellow, fine ................. $\quad .40$

GOLD THROAT (K). Blood red and gold, odd............. 10

GOLDEN GLEAM (K). Very goor light orange prim... . 20

GLADNESS (K). Unusual lavender prim. orchid.......... .15

GOLDEN SWALLOW (A). Another yellow................. 15

GOLDEN SALMON (K). Just it and fine................... 3.00

H. C. GOEHL (F). Large white, crimson blotch.......... .30

HARBINGER (Sanford) New. Orange.................... 5.00

HERADA (A). Pure mauve, a beauty ......................... $\quad .10$

HELGA (K). Early salmon rose. Light throat............... 08

HELEN WILLS (S). New Superb white.................. 10.00

HENRY FORK (D). Large purple.............................. .35

INDIAN MAID (K). Peach blossom pink. Very fine tall and showy. True variety. Not Mrs. Schweppe which was sent out as such.....

INDIAN SUMMER $(\mathbf{K})$. Late, large light lavender....... ..50

ILLUMINATOR $(\mathbf{H})$. Tall deep rose red, purple lines.. .25

IMMENSITY $(\mathbf{K})$. Excellent large bright salmon ........ .25

J. A. CARBONE (D). Orange salmon, yellow throat ... . .25

JACOBA VAN BEIJEREN (H). Light self purple........ .25

JENNY LIND (Dec.). Light salmon, cream throat....... .15

JOE COLEMAN (K). Ruffled red, different, good......... .05

J. T. PIRIE (K). Mahogany brown, cream throat........ $\quad .20$

JOERGS WHITE. Very large excellent white............. 2.00

JOHN ALDEN $(\mathbf{F})$. Fine yellow with red throat.......... .50

JUNO (C). An orange prim. of Colemans.................. .10

JEWELL $(\boldsymbol{Z})$. Light salmon, yellow throat, fine............. .08

J. T. McCUTCHEON (K). Large deep rose................ 2.00

KIRCHHOFES VIOLET. Best dark blue, excellent.... . .25

LE MAERCHAL FOCH (H). Early light pink............ .05

LILAC WONDER (Eu.). A real fine new Glad. Medium bulbs

LONGFELLOW (Dec.). Large La France pink, great.

LOS ANGELES (Hyd.). Delicate pink, cerise feather.

LOUISE (W). Bright lavender, wine throat, good..........

LUSTRE (K). Dark orange vermillion. Grand red........

LILAC OLD ROSE $(\mathbf{K})$. Very attractive prim............. .25

MARNIA (K p.). Beautiful golden orange.................. 1.00

MASTERPIECE $(\mathbf{K})$. American beauty rose................ 15

MAURICE FULD (G). Large beautiful rose pink ......... 1.00

MADISON COOPER (K). Fine dark garnet red............ Sold

MARIE KUNDERD (K). Best early white, ruffled....... .25

MARIETTA (M). Large salmon-tinged orange................ .35

MARSHAL FOCH (K). Large salmon pink, late........... $\quad .10$

MARY PICKFORD (K). Early white, yellow throat.... $\quad .10$

MISS. SPOKANE (K). Strong dark salmon, fine........... .15

MISS. MADISON (By.). Clear delicate pink .................. $\quad .40$

MRS. DR. NORTON $(\mathbf{K})$. Cream and pink, none better.

MRS. F. C. PETERS (F). Like a cattleya orchid..

MRS. F. PENDELTON (K). Shell pink, crimson blotch

MRS. J. R. WALSH (D). Flesh pink, red blotch.

MRS. WM. E. CLARK (F). Salmon pink, red blotch 
MRS. H. E. BO'THIN (D). Ruffled flesh salmon, blotch $\quad .10$

MRS. G. W. MOULTON (K). Giant violet red.......... . 05

MRS. KR. PRESTGARD (Dec.). New pure white, good

MRS. L. S. SCHWEPPE (K). Peach blosson pink

MRS. LEON DOUGLAS (D). Begonia rose and scarlet

MRS. M. J. KILLION (F). Excellent white

MRS. VAN KONYNENBURG (H). The best light blue medium bulbs

MME. MOUNET-SULLY (De G). Creamy white, blotch

Sold

MURIEL. An excellent so called blue............................

MYSTIC (K). Flaked rose pink, white throat, fine...... 15

M YRTLE (K). Early arbutus pink and white............. .05

M YRA $(\mathbf{K})$. Large pink and yellow prim....................... 05

MING TOY $(\mathbf{K})$. Beautiful buff and yellow, superb..... $\quad .20$

MIDSUMMER DREAM (K). Red prim. of quality..... .15

MAGIC (K). Lavender blue, purple throat................... 10

MRS. P. W. SISSON (G). Creamy light pink ........... 10.00

MALLOW (St.). Large creany white.......................... 1.00

MARCONI (Dec.). Rose purple, aster purple throat..... $\quad .50$

MRS. F. C. HORNGERGER. Best new white.......... 5.00

NORMA TALMADGE (Dec.). Large clear sulphur yellow.

OBELISQUE (H). Large cream, yellow lip.

1.00

OPALESCENT (B). Pale lavender....................... .25

ORANGE FLAME (K). Ruffled vermillion scarlet, fine .75

ORANGE QUEEN (H). Beautiful orange yellow.......... .25

POLAR STAR (M). Very pretty ruffled white............. . 20

PRIORITY (B). One of his popular pinks.................... 25

PARAMOUNT $(\mathbf{K})$. Choice pink and cream...................... .25

PEACH ROSE $(\mathbf{K})$. Beautiful clear rose pink.............. .30

PINK LIL Y $(\mathbf{K})$. Ruffled deep rose pink, fine............. .15

PURPLE GLORY (K). Deep velvety maroon.............. .20

PRIDE OF LANGASTER (K). Ruffled orange.............. 05

PRIDE OF GOSHEN (K). Ruffled flesh pink ............... 05

POLLYANNA (Dec.). Very good yellow ...................... 15

PYTHIA (K). Large channel red, great ........................ Sold

PRIDE OF WANAKAH (Cr.). Deep lavender rose....... .75

PRINCEL Y (Kp.). A fine new white........................ 1.00

PURPLE BU'TTERFLY (Gr.). Very pretty................ .50

PURPLE QUEEN (K). Another well liked ................... 2.00

QUIN'TON (Kp.). Early coral pink, yellow lip............ $\quad .40$

RITA BECK $(\mathbf{F})$. Snappy light pink, grand ................ 10.00

ROSEMARY (Ba.). Pink with lavender lines.............. .75

REMEMBRANGE (K). Choice salmon rose................ 15

RUFFLED AMERIGA (K). Lovely light pink............ . 25

RED FIRE (K). Magnificent fiery red ........................ . 20

RED COPPER (K). Deep salmon rose flaked blue.......... .15

RICHARD DIENER (D). Rose salmon, yellow center.. $\quad .25$

ROSE MIST (F). O!d rose, edged gray..................... 1.00

ROSE ASH (M). Tall ashes of roses, popular................

ROSE GLORY (K). Pure rose pink, ruffled............. $\quad .10$

ROSALIND $(\mathbf{K})$. Dark aniline red, very fine............... .30

ROSELLA. Near old rose, very well liked..................... 15

RU'TH HUN'TINGTON (K). Ruffled pale lilac, tail..... . . 20

R. J. KUNDERD (K). Large orange vermillion.............. .25

R. V. K. (H). Large rose, yellow throat....................... 05

ROMANGE (K). Salmon rose bordered blue, odd .......... .20

SALMON GLOW. Hornbergers fine salmon and yellow 1.50

SARABAND (S). Rich bordeaux, straw yellow throat .. 1.00

SVEA $(\mathbf{K})$. Dainty salmon pink, canary yellow throat .. $\quad .15$

SCARLANO $(\mathbf{K})$. Dazzling orange scarlet, fine........... $\quad .10$

SCARLET WONDER (Gr.). Very large clear red.......... .20

SCARLET PRINGEPS $(\mathbf{K})$. One of the fine Glads....... .10

SHEILA (G). Beautiful large light salmon.................. $\quad .10$

SHEILAS SISTER (G). Similar, but larger................... 05

SHELL PINK (K). Very fine flower, popular............... $\quad .20$

SEABROOK GEM (R). Light rose lavender.............. . 10 
SIDNEY PLUMMER (S). Soft yellow, amber lip......... .25 SANS PARIEL. A fine apricot.................................. .25 SHARON ROSE (C). Large, beautiful, distinctive...... .35 SWEET LAVENDER (C). Early light lavender, fine..... $\quad .15$ SNOW GLOR Y $(\mathbf{K})$. Pure white, iris blue lines........... .25 SUPERBA (C). Large carmine red................. .40 SULPHUR GLOW $(\mathbf{K})$. Very popular with visitors..... .15 SPLENDORRA $(\mathbf{K})$. Deep wine black, very fine .......... .20 SUNNYMEDE $(\mathbf{F})$. Beautiful orange yellow................... 35 SULPHUR FRILLS (K). Sulphur yellow, ruffled.......... .50 SONIA $(\mathbf{K})$. Very bright red prim., well liked................ .05 SALMON BEAU'TY $(\mathbf{K})$. Deep salmon, yellow throat.. .08 SALMON BUFF (K). Attractive prim. of quality......... .10 SNOW BOY (K). Tall white purple blotch, good.......... $\quad .10$ SOUVENIR $(\mathbf{H})$. Deep yellow prim.......................... 05 TARO $(\mathrm{K})$. Fine American Beauty ............................. .20 TREASURE $(\boldsymbol{Z})$. Best deep yellow prim....................... 15 THOTH $(\mathbf{K})$. Beautiful white prim., yellow throat........ $\quad .10$ TOPAZ (K). Salmon pink and buff............................ 05 THISTLE $(\mathbf{K})$. Very good rose red, ruffled.................. .15 TWILIGHT $(\mathbf{K})$. Creamy buff, yellow and pink, fine... . 20 TYREAN BEAUTY $(\mathbf{K})$. American Beauty shade, large $\quad .10$ TYCKO ZANG (A). Salmon pink, immense, lt. throat 1.50 UHLAN CHIEF (K). Bright vermillion scarlet, fine.... .15 ULRICA $(\mathbf{K})$. Fine yellow and orange prim................. .05 VIOLET BEAUTY $(\mathbf{K})$. Tall violet, deeper throat...... .05 VIOLET GLORY $(\mathbf{K})$. Deep self violet....................... 10

WHITE WONDER (Kp.). Large good white................ .05

WILBRINCK $(\mathbf{H})$. Early light pink .......................... 05

WHITE GLOR Y $(\mathbf{K})$. White, iris blue throat............. 10

WM. KENT (D). Creamy shell pink and yellow........... $\quad .70$

WM. BADGER (M). Large salmon rose, cream throat .50

W. E. FRYER (K). Tall deep violet, fine ................ 10

W. H. PHIPPS (D). Twenty or more light rose salmon blooms open at a time.

WANETA (K) Rose pink, cream throat, fine $(\mathbf{K}$............. 1.00

YELLOW TREASURE (A). Fine yellow prim............... .10

YELLOW WONDER (K). His best yellow.................. 10.00

YOUELLS FAVORITE $(\mathbf{K})$. Rosy lavender pink, late an old one but good just the same.
(A) Austin
(B) Glad Bill
(Bet.) Betscher
(C) Coleman
(F) Fischer
(H) Holland
(Gr.) Groff
(K) Kunderd
(Kp.) Kemp
(S) Salbach
(By.) Boynton
(D) Diener
(G) Gage
(H) Hinkel
(L) Lemoine
(M) Metzner
(Z) Zeestraten

Dozen Bulbs at 10 times single rate. Half and quarter dozen at Dozen rate. Bulbs by 100 at seven times dozen rate. 50 bulbs at 100 rate.

Half size bulbs at $60 \%$ of large price. Plant different sizes and extend blooming season.

Bulblets; one dozen for price of one large bulb. Special prices on larger quantities. For bulk see wholesale list.

In some instances prices are lower. than those quoted at Field and at Shows. All such cases extra bulbs will be added to order. Where price has advanced no change will be made.

If in doubt about choosing varieties send the amount you wish to spend and I will send you a varied collection sure to please. I fill many orders this way.

Making a present to someone? Send them a nice "GIFT BOX" of Glad. Bulbs. 